\title{
STUDIES OF SOLIDS AND ADSORBATES IN SYNCHROTRON RADIATION EXCITED SOFT X-RAY FLUORESCENCE
}

\author{
J. NORDGREN AND N. WASSDAHL
}

Uppsala University, Department of Physics, Box 530, 75121 Uppsala, Sweden

A progress report is presented on recent developments in soft X-ray emission spectroscopy using synchrotron radiation. Some key experiments are discussed demonstrating that new information can be gained by using synchrotron radiation for this purpose. Recent work on highly resolved soft $\mathrm{X}$-ray emission from monolayers of adsorbed species is commented on and some perspectives are given regarding further developments expected from the third generation sources that are underway.

PACS numbers: 78.70.En

\section{Introduction}

The last decade's developments in the field of synchrotron radiation sources have opened new and interesting possibilities to pursue soft X-ray emission spectroscopy (SXES). Although SXES was an established method for band structure studies of metals, alloys and other systems already a couple of decades ago [1], the advantages offered by synchrotron radiation have been sufficient to speak of a renaissance for this method. Already some ten years ago white light synchrotron radiation was used to excite SXES spectra [2], but at that time it was not possible to achieve experimental conditions allowing monochromatized excitation.

A few properties of synchrotron radiation are of particular value in the context of soft X-ray emission. Firstly, the very fact that this excitation agent involves photons rather than electrons makes it possible to obtain a higher degree of selectivity in the excitation since one a voids the low energy tail of inelestically scattered excitation electrons. Also, larger penetration depths for photons than for particles of corresponding energy allows investigation of bulk properties in solids. A further consequence is the fact that photons allow more chemically fragile samples to be studied, an aspect of importance for SXES, since it is a "low-intensity method", often requiring substantial data accumulation times.

Secondly, synchrotron radiation, as compared to other X-ray sources, offers high brilliance, i.e. both high intensity and low emittance. This is very important for soft $\mathrm{X}$-rays, since soft $\mathrm{X}$-ray optics are of low acceptance due to the requirement 
of grazing angles to attain total reflection. High brilliance then is a prerequisite in order to obtain a sharply monochromatized excitation beam of high intensity, which, in turn, is required to make full use of soft X-ray emission spectroscopy. One should bear in mind that the fluorescence yielded in this energy range is typically only $0.1 \%$ [3] which puts further demands on the power of the excitation source as well as on the efficiency of the recording instruments.

A third property of synchrotron radiation that is attracting an increasingly growing interest for soft $\mathrm{X}$-ray spectroscopy is its polarization properties. For other spectroscopic methods like photoemission and X-ray absorption the well-defined polarization of synchrotron radiation has been extensively used, and it has also been introduced as an important element in X-ray emission spectroscopy [4].

\section{Selective excitation of SXES spectra}

One key feature of synchrotron radiation excited SXES is of course the energy selectivity of the excitation. X-ray satellites, notoriously accompanying the diagram lines in X-ray emission spectra, were observed and to certain extent understood a long time ago [5]. In many cases, especially for metallic systems where screening is efficient, the X-ray satellites tend to overlay the diagram lines in a way which obscures the main features and obstructs proper analysis. One remedy for this is of course a more energy selective excitation since a great deal of the $\mathrm{X}$-ray satellites originate in multiple excitation/ionization processes.

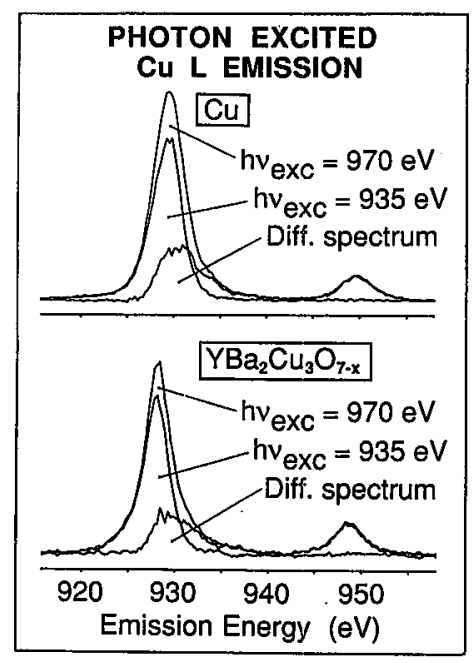

Fig. 1. Copper $L_{2,3}$ emission spectra of $\mathrm{Cu}$ metal (above) and $\mathrm{YBa}_{2} \mathrm{Cu}_{3} \mathrm{O}_{7-x}$ (below), excited at energies near the $L_{3}$ threshold and well above the $L_{2}$ threshold, respectively. The difference spectrum is also plotted.

Separation of satellite structure by selective excitation is demonstrated in Fig. 1 where the $\mathrm{Cu} L$ emission spectra of metallic copper and the $\mathrm{YBa}_{2} \mathrm{Cu}_{3} \mathrm{O}_{7-x}$ 
superconducting oxide are shown [6]. One notices that reducing the excitation energy from $970 \mathrm{eV}$ to $935 \mathrm{eV}$, i.e. to just above the $L_{3}$ threshold, makes the $L_{2}$ line disappear, as expected, but it also leads to a different form of the $L_{3}$ spectrum. This difference comes about from the removal of the satellites that are caused either by shake up/shake off processes in the $L_{3}$ excitation or by Coster-Kronig decay of $L_{2}$ vacancies, both processes being energetically forbidden at the lower excitation energy. In the local one electron approximation the threshold excited $L_{3}$ spectrum then reflects the "clean" $3 d$ partial density of states. Forming the difference between the " $970 \mathrm{eV}$ spectrum" and the " $935 \mathrm{eV}$ spectrum" yields the pure satellite spectrum, as seen in the figure. One notices, incidently, comparing the satellite spectra of the metal and the oxide, that they are different, presumably reflecting a quenching of the Coster-Kronig decay of $L_{2}$ holes induced by the modification of the valence band in forming the oxide.

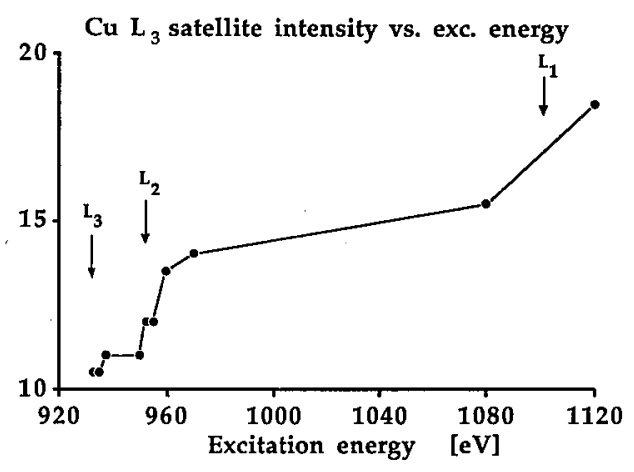

Fig. 2. Variation in $\mathrm{Cu} L_{3}$ satellite intensity vs. excitation energy.

In Fig. 2 the $\mathrm{Cu} L_{3}$ satellite intensity has been plotted against excitation energy. One observes a gradual increase of the intensity between the energy thresholds of the subshells, whereas at the thresholds, in particular the $L_{2}$ threshold, a rapid increase occurs due to the Coster-Kronig decay of the $L_{2}$ hole state which leads to $L_{3} M$ double hole states, i.e. initial states for satellite transitions.

By recording emission spectra at various excitation energies from threshold to high excess energies the satellite spectra can be studied in considerable detail. Figure 3 shows the $L_{2,3}$ emission spectrum of $\mathrm{Zn}$ metal, excited at a variety of energies from near the $L_{3}$ threshold up to and above the $L_{1}$ threshold. One can clearly observe how the satellite structure on the high energy side of the $L_{3}$ peak is growing up. Just above the $L_{3}$ threshold the only possible excitation is that of a single $L_{3}$ hole, whereas at higher energies the $L_{3}$ excitation can be accompanied by additional $M$ electron excitations, giving rise to the satellites [7].

The usefulness of tunable excitation of X-ray emission is not restricted to the separation or studies of $\mathrm{X}$-ray satellites. The ability to tune the excitation energy to certain resonance energies facilitates studies of the dynamics of excitation processes and it can help to focus on details in the electronic structure. Figure 4 shows $O K$ emission spectra of a few different copper oxides, exhibiting quite different features. 


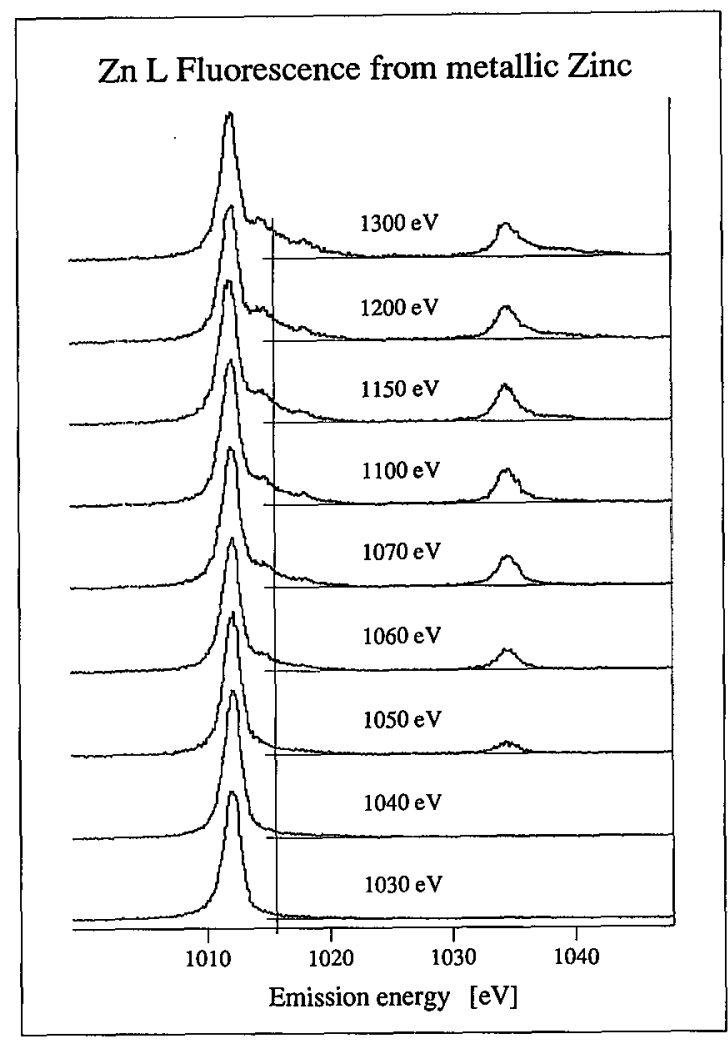

Fig. 3. $\mathrm{Zn} L_{2,3}$ emission spectra of $\mathrm{Zn}$ metal excited at a variety of energies between the $L_{3}$ and the $L_{1}$ thresholds.

Two of the samples studied were, again, 1-2-3 superconductors differing slightly in oxygen content, leading to a $1.3 \mathrm{~K}$ difference in critical temperature. For a wide range of excitation energies, and in particular for non-selective excitation, these two samples show almost identical oxygen $K$ emission spectra. At certain distinct energies, though, as for example at $541 \mathrm{eV}$, which was the energy used in this case, a dramatic difference between the spectra is observed also between these two samples. This sensitivity of the X-ray spectrum to the crucial oxygen content may provide useful information for the understanding of these systems.

In one of the early experiments using tunable photon excitation of SXES spectra the $\mathrm{N} K$ and $\mathrm{Ti} L$ emission of TiN were studied [8]. By tuning the excitation energy below the Ti $2 p$ threshold the $\mathrm{N} K$ spectrum could be recorded for the first time without the accidentally overlapping inner-core transition in Ti $(3 s-2 p)$, see Fig. 5, bottom. The middle spectrum in the figure shows the Ti $L$ emission excited just above the $L_{3}$ threshold, consequently showing the bare $L_{3}$ contribution to the spectrum. Taking into account the $6 \mathrm{eV}$ spin-orbit splitting between the $L_{2}$ and the 


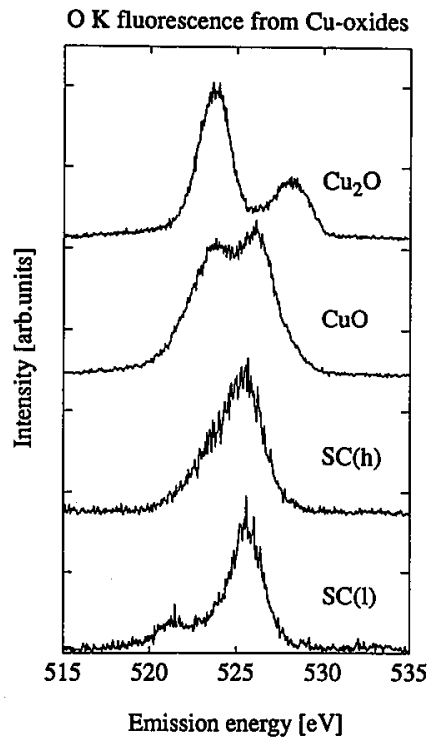

Fig. 4. $\mathrm{O} K$ emission spectra of various copper oxides, among them two $\mathrm{YBa}_{2} \mathrm{Cu}_{3} \mathrm{O}_{7-x}$ samples with slightly different oxygen content, marked $\mathrm{SC}(\mathrm{l})$ for lower content and $\mathrm{SC}(\mathrm{h})$ for higher. The emission was excited at $541 \mathrm{eV}$ for all samples.

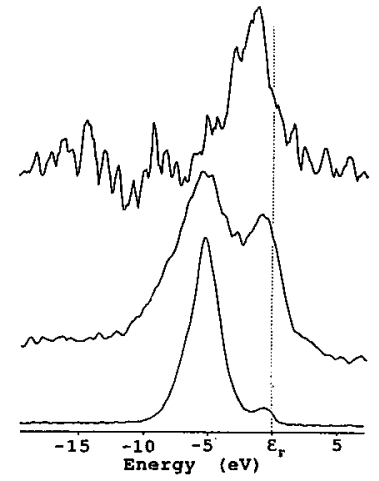

Fig. 5. Soft X-ray fluorescence spectra of TiN. Bottom, $\mathrm{N} K$ emission excited by $410 \mathrm{eV}$ photons. Middle, Ti $L$ emission excited at $458 \mathrm{eV}$. Top, difference spectrum between $\mathrm{Ti}$ $L$ emission excited at $480 \mathrm{eV}$ and $458 \mathrm{eV}$, respectively. The spectra have been aligned by means of XPS data.

$L_{3}$ states an apparent agreement with previously reported, non-selectively excited spectra was thus observed. However, the falseness of this agreement was realized by comparing the $L_{2}$ and the $L_{3}$ emission, as can be done by looking at the uppermost spectrum where the derived $L_{2}$ emission spectrum is shown (derived by forming 
the difference between spectra excited above and below the $L_{2}$ threshold). One notices that the two spectra are in fact totally different, and therefore the line at $460 \mathrm{eV}$ had to be given a different explanation than the previously excepted one.

\section{Studies of surface adsorbates}

The large information depth offered by the comparatively deep penetration of X-rays, as compared to electrons, is a useful asset whenever bulk properties are being studied. However, this does not mean that soft X-ray emission cannot be used in the study of extreme surface problems. Detection of light elements in low resolution SXES is being successfully pursued in surface physics experiments [9], and as a matter of fact, it has recently been shown [10] that even highly resolved soft X-ray fluorescence spectra can be obtained from fractions of monolayers of adsorbed atoms and molecules on surfaces by using grazing incidence excitation. Figure 6 shows oxygen $K$ emission spectra of a $\mathrm{Ni}(100)$ surface exposed to respectively $50 \mathrm{~L}$ and $1000 \mathrm{~L}$ of oxygen. In the former case a c $(2 \times 2)$ monolayer adsorbate structure is formed, giving rise to a spectrum with a distinct Fermi edge, directly reflecting the hybridization between the $\mathrm{O} 2 p$ and the $\mathrm{Ni} 3 d$ electrons. In the latter

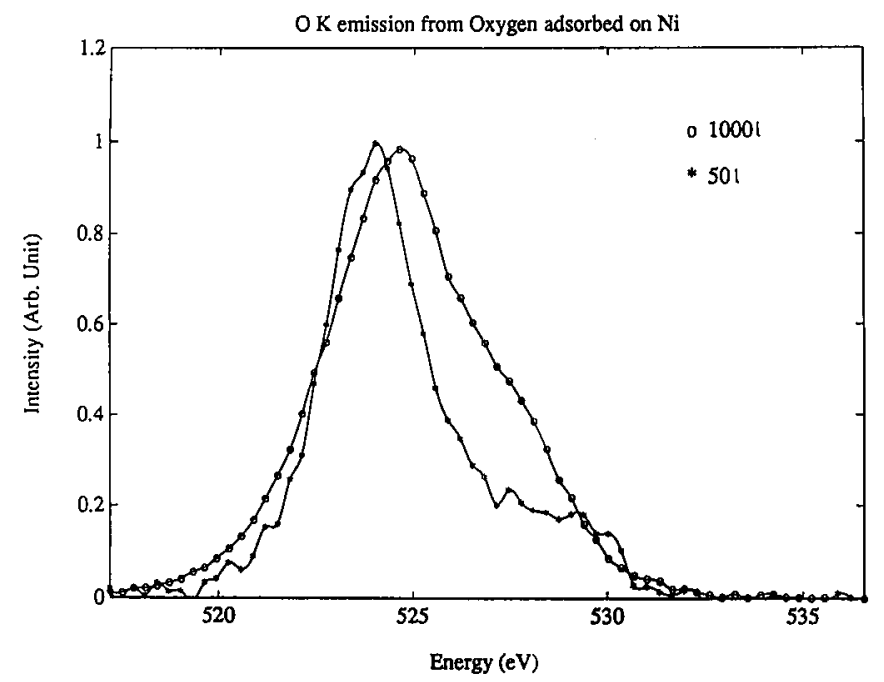

Fig. 6. O $\mathrm{K}$ emission spectra, excited with white light synchrotron radiation from a $\mathrm{Ni}(100)$ surface exposed to $50 \mathrm{~L}$ and $1000 \mathrm{~L}$ of oxygen, respectively.

case, the high exposure forces $\mathrm{NiO}$ to be formed rather than only an adsorbate monolayer, and indeed a different spectrum is observed, which is characteristic of $\mathrm{NiO}$. To the present a number of different adsorbate systems have been studied, including the frequently investigated $\mathrm{CO} / \mathrm{Ni}(100)$, yielding results which indicate that there may be very interesting prospects for SXES in surface physics research, especially with the even higher brightnesses expected from the third generation synchrotron radiation sources. Obviously the ability of SXES to probe the local 
partial density of states is of great value in this kind of study, since one escapes the difficulty to separate the adsorbate derived states encountered in photoemission experiments. The method then allows a direct means to investigate the bonding of the adsorbate in terms of orbital hybridization. It could also be mentioned in this context that the comparative "softness" of photon excitation compared to electrons is an important feature without which studies of this kind would be very difficult.

A direct effect of alignment is observed in the $\mathrm{O} K$ emission spectrum of $\mathrm{CO}$ adsorbed on $\mathrm{Ni}(100)$, where it turns out that the $\sigma$-orbitals do not contribute to the emission spectrum in the direction of the surface normal, as expected considering the perpendicular bonding geometry of this system and the dipole intensity distribution [10]. Furthermore, given the ability to record soft X-ray emission spectra from submonolayer systems it is clear that the proper use of the polarization of the synchrotron radiation used for excitation adds an important element of selectivity. Such studies have not been made yet, but are being planned for the near future.

\section{Experimental considerations}

A main characteristic feature of soft $\mathrm{X}$-rays is the very strong absorption in matter. As opposed to both shorter and longer wavelengths of the electromagnetic spectrum soft $\mathrm{X}$-rays can only penetrate windows of submicron thickness, a condition which has considerable experimental implications. The soft $\mathrm{X}$-ray range has its short wavelength limit around $2 \AA$ where the radiation starts to penetrate macroscopic distances at atmospheric pressures. The long wa velength limit is more floating, but taking it as the softest X-ray transition (Na $L$ ) puts it near $400 \AA$.

A subrange of the soft X-ray range is the ultra-soft X-ray range (USX), defined by taking the short wavelength limit to be about $10 \AA$, i.e. ending the range at $1 \mathrm{keV}$ photon energy rather than $5 \mathrm{keV}$. There are some reasons for making this subdivision, mainly of experimental nature. The greater part of the $\mathrm{X}$-ray range is accessible for spectroscopy by means of instruments based on Bragg diffraction. IIowever, below the $1 \mathrm{keV}$ region the lack of suitable crystals and the increasing absorption present problems which have made this part of the spectrum less accessible. Especially below the oxygen $K$ edge the power of the Bragg technique is limited due to the lack of natural crystals (this condition may change in the future when sufficiently thick and high quality synthetic multilayer structures become a vailable as dispersive elements). Grating diffraction, on the other hand, which is the commonly used technique in spectroscopy for visible and vacuum ultraviolet wavelengths, loses power going to very short wavelengths due to decrease in efficiency and resolution. It seems that for many experiments the crossing point occurs around $1 \mathrm{keV}$ at which the other method becomes more advantageous.

Focusing grating instruments are often the only choice for high resolution USX emission spectroscopy (USXES), especially in connection with sources of limited extension, such as often encountered with synchrotron radiation. Then the advantage offered by grating diffraction of simultaneously dispersing all wavelengths, as opposed to Bragg reflection, can be fully utilized provided that multichannel detection is employed. Going one step further and arranging detection 
in two dimensions offer increased sensitivity without significant losses in resolution. This is because the part of the astigmatic imaging errors associated with the curvature of the spectral lines can be accounted for, allowing larger acceptance in the perpendicular plane. In other words, the recording of long, slightly curved spectral lines can be done without loosing resolution appreciably. Below is given a brief presentation of a soft X-ray spectrometer for high resolution work, which has played a key role in recent progress in soft X-ray spectroscopic studies of electronic structure. A more detailed description of the instrument can be found in Ref. (11).

The basic idea behind the design of this instrument is the use of several fixed spherical gratings and a large position sensitive detector, see Fig. 7. The gratings

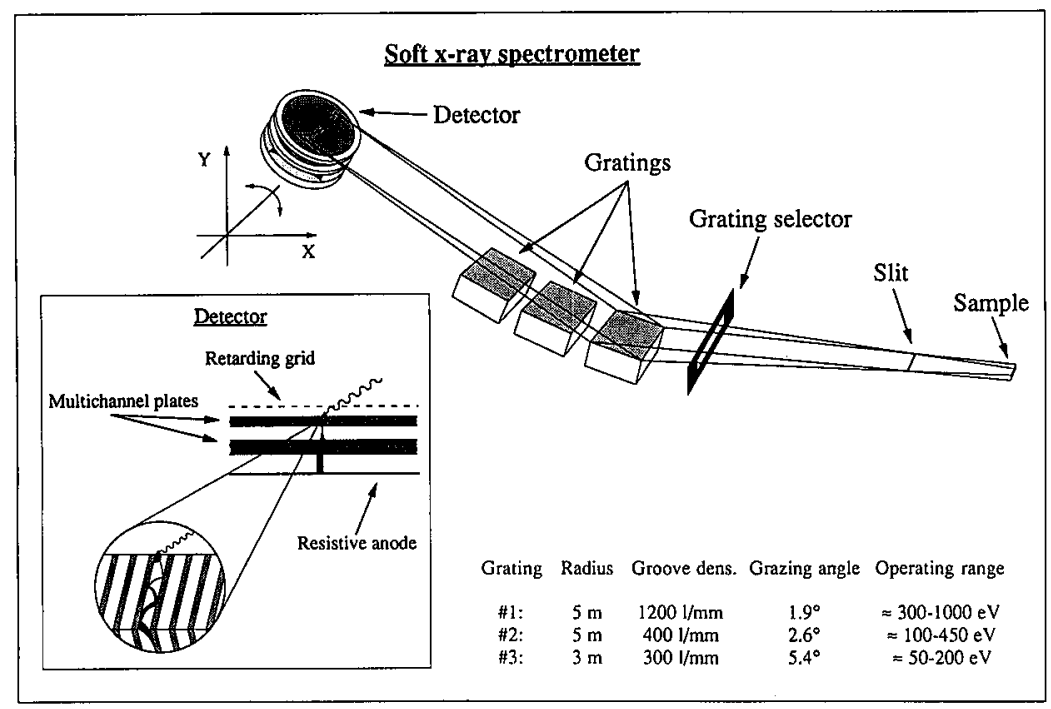

Fig. 7. Principles of a multi-grating grazing incidence spectrometer used for synchrotron radiation excited fluorescence spectroscopy.

have different radii and groove densities chosen in order to optimize the performance over a wide wavelength range and they are working at different incidence angles chosen to match the respective wavelength range covered. The detector can be positioned tangential to the Rowland circle of the respective gratings by precision movement in a three-axis coordinate system (two translations, one rotation). This arrangement allows some important qualities to be attained. The resolution and luminosity are high and the instrument size can be brought down to a minimum. Simple flange-mounting is feasible, whereby one can conveniently use the instrument at different sources. Another advantage is the freedom of configuring the instrument with different gratings in order to optimize performance for a particular wavelength region.

The luminosity of the instrument is high owing to the use of a large efficient detector and the multi-grating arrangement which keeps the distance between source and detector small. The detector is based on $50 \mathrm{~mm}$ multichannel plates 
(MCP) and two-dimensional read-out is made by means of resistive anode technique. The efficiency of the detector is enhanced by coating the front MCP with a layer of CsI and an entrance electrode is introduced in order to capture electrons ejected from the interstitial surfaces of the MCP, which are the active surfaces at grazing incidence. Holographic ion-etched gratings have been used offering typical diffraction efficiencies of $10 \%$.

Most of the work presented in this review was made using monochromatized synchrotron radiation for the excitation. For this work a 32-pole wiggler in one of the straight sections of the DORIS storage ring at DESY in Hamburg was used. A plane grating monochromator (FLIPPER I) was used for wavelength selection in the range $20 \mathrm{eV}$ to $1500 \mathrm{eV}$ [12]. The monochromator has a number of different premirrors, which, one at the time, can be put into position in order to illuminate the grating at the most suitable angle for the desired wavelength range. The source point is at about $35 \mathrm{~m}$ away from the monochromator making the beam nearly parallel, and a parabolic mirror is used as the postmirror to focus on the exit slit of the monochromator. The output flux is of the order of $10^{13}$ photons per second at a bandpass of about $0.5 \%$. These high flux levels are necessary due to the low fluorescence yield for ultra-soft X-rays and the limited solid angle of acceptance for grazing incidence spectrometers.

An important point concerning the detection system of the present instrument in connection with the use of storage ring sources is related to the time structure of the ring. The duty cycle of such sources are normally quite low which may allow the electronic noise of the detection system to be virtually eliminated by synchronizing the detector with the time structure of the ring. This is particularly true for a few-bunch storage ring like DORIS. Using standard electronics (ECL), obtaining a time window' of $7 \mathrm{~ns}$ the dark noise was reduced to less than $3 \%$ in the 4-bunch mode used for dedicated synchrotron radiation operation of DORIS.

A slightly different experimental approach was taken to pursue SXES by one group who used toroidal gratings in their recording instrument and a transmission grating monochromator operating at a bending magnet beamline at NSLS in Brookhaven [13]. Significant contributions to this field have been made by this group, especially in the low energy part of the soft X-ray,range, e.g. in the study of excitation energy dependence for SXES of Si and solid noble gas systems [14].

\section{Future prospects}

One could easily anticipate further possibilities in using soft X-ray emission spectroscopy in connection with the new third generation synchrotron radiation sources presently being constructed at various locations in the world. Experiments that have been demonstrated just feasible at the present facilities will be made on a routine basis and again new experiments which are presently beyond reach due to lacking performance will become possible. Ultimately, though, there are limitations put by e.g. maximum doses of exposure for certain fragile systems and unless completely new and more efficient methods for registration of highly resolved emission spectra are found, there will be a lower sensitivity of soft X-ray emission than e.g. Auger emission spectroscopy also in the future. 
One exciting prospect is the ability to excite SXES spectra of organic molecules at excitation bandpasses of $100 \mathrm{meV}$ and below. This would allow separation of emission spectra pertaining to chemically shifted identical species. Using non-selective excitation the different spectra will appear overlaid leading to smearing out and obstruction of detailed analyses, as shown in Fig. 8 depicting the X-ray emission from aniline [15]. The $\mathrm{N} K$ emission shows separated bands, whereas the C $K$ emission consists of four different superimposed spectra slightly shifted with respect to each other. If separation of these spectra would be possible, one could

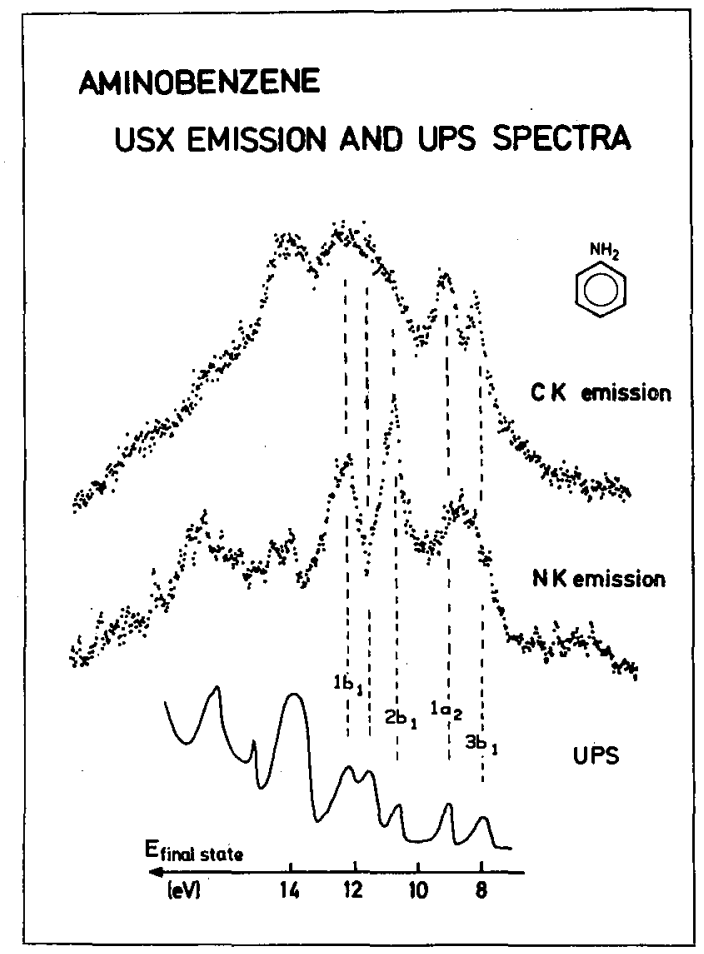

Fig. 8. N $K$ and $\mathrm{C} K$ emission spectrum of gas phase aniline (aminobenzene), excited by $8 \mathrm{keV}$ electron impact.

fully exploit the ability of SXES to probe the valence electronic structure. Such narrow excitation would also open ways to perform detailed studies of surface effects and even buried layers and interfaces, such as, for example, studies of the bonding of polymer layers to metal surfaces.

Having access to sufficiently narrow excitation may also offer possibilities to study coherent processes in $\mathrm{X}$-ray emission. One example is the lifetime-vibrational interference observed in small molecules where the vibrational splitting and the core state lifetime width are of comparable magnitude [16]. Another example is a possible coherent contribution recently observed in the $\mathrm{C} K$ emission spectra of diamond excited from threshold to $30 \mathrm{eV}$ above threshold [17]. 
Generally, the use of polarization and angular resolved SXES is expected to provide further assets to this spectroscopic method, and an experiment station for angular resolved soft $\mathrm{X}$-ray fluorescence spectroscopy using monochromatized synchrotron radiation has recently been built for installation at the Bypass laboratory at the DORIS storage ring. One goal set out in this project is to study angular resolved $L$ emission spectra from $3 d$ transition element systems, thereby obtaining m-resolved $3 d$ decomposition of the valence band. A further prospect in this connection is the use of circularly polarized synchrotron radiation for the excitation of SXES spectra in order to allow detailed studies of magnetic properties also in emission which may lend itself to valuable comparison with calculated ground state properties.

\section{Acknowledgement}

This work has been supported by the Swedish Natural Science Research Council. We would like to express our gratitute to various people who have contributed to the work presented in this paper. Among them are G. Bray, P. Glans, R. Nyholm and J.-E. Rubensson.

\section{References}

[1] See e.g. D.J. Fabian (Ed.), Soft X-Ray Band Spectra, Academic Press, New York 1968.

[2] N. Kosuch, E. Tegeler, G. Wiech, A. Faessler, Nucl. Instrum. Methods Phys. Res. 152, 113 (1978).

[3] M.O. Krause, J. Phys. Chem. Ref. Dala 8, 307 (1979).

[4] S.H. Southworth, D.W. Lindle, R. Mayer, P.L. Cowan, Phys. Rev. Lett. 67, 1098 (1991).

[5] G. Wentzel, Z. Phys. 31, 445 (1925); M.J. Druyvesteyn, ibid. 43, 707 (1927).

[6] N. Wassdahl, Thesis, Uppsala University, Acta Universitatis Upsaliensis, 114 (1987).

[7] N. Wassdahl, G. Bray, S. Cramm, P. Glans, P. Johansson, R. Nyholm, N. Mårtensson, J. Nordgren, Phys. Rev. Lett. 64, 2807 (1990).

[8] J.-E. Rubensson, N. Wassdahl, G. Bray, J. Rindstedt, R. Nyholm, S. Cramm, N. Mårtensson, J. Nordgren, Phys. Rev. Lett. 60, 1759 (1988).

[9] D.A. Fischer, J. Colbert, J.L. Gland, Rev. Sci. Instrum. 60, 1596 (1989).

[10] N. Wassdahl, A. Nilsson, T. Wiell, H. Antonsson, L.C. Duda, J.H. Guo, N. Mårtensson, J. Nordgren, J.N. Andersen, R. Nyholm, submitted for publication.

[11] J. Nordgren, G. Bray, S. Cramm, R. Nyholm, J.-E. Rubensson, N. Wassdahl, Rev. Sci. Instrum. 60, 1690 (1989).

[12] F. Senf, K. Berens v. Rautenfeldt, S. Cramm, C. Kunz, J. Lamp, V. Saile, J. Schmidt-May, J. Voss, Nucl. Instrum. Methods Phys. Res. A 246, 314 (1986).

[13] T.A. Callcott, K.-L. Tsang, C.H. Zhang, D.L. Ederer, E.T. Arakawa, Rev. Sci. Instrum. 57, 2680 (1986).

[14] See e.g. J.-E. Rubensson, D. Mueller, R. Shuker, D.L. Ederer, C.H. Zhang, J. Jia, T.A. Callcott, Phys. Rev. Lett. 64, 1047 (1990); J.J. Jia, W.L. O'Brian, T.A. Callcott, Q.Y. Dong, J.-E. Rubensson, D.R. Mueller, D.L. Ederer, Phys. Rev. Lett. 67, 731 (1991). 
[15] J. Nordgren, L. Selander, L. Pettersson, R. Brammer, M. Bäckström, C. Nordling, Chem. Phys. 84, 333 (1984).

[16] A. Flores-Riveros, N. Correia, H. Ågren, L. Pettersson, M. Bäckström, J. Nordgren, J. Chem. Phys. 83, 2053 (1985).

[17] Y. Ma, N. Wassdahl, P. Skytt, J. Guo, J. Nordgren, P.D. Johnson, J.-E. Rubensson, T. Böske, W. Eberhardt, S.D. Kevan, to be submitted for publication. 